\title{
ANÁLISIS PROSPECTIVO DE LA INDUSTRIA DE DESARROLLO DE SOFTWARE EN COLOMBIA
}

\author{
Jessica Piña Taborda \\ Facultad de Negocios, Gestión y Sostenibilidad \\ Especialización en Gestión Empresarial \\ jecapina@gmail.com \\ Diana Marcela Castaño Ospina \\ Facultad de Negocios, Gestión y Sostenibilidad \\ Especialización en Gestión Empresarial \\ diana.578@hotmail.com \\ Leonardo Enrique Hernández Díaz \\ Facultad de Negocios, Gestión y Sostenibilidad \\ Especialización en Gestión Empresarial \\ Juan David Garro Torres \\ Facultad de Negocios, Gestión y Sostenibilidad \\ Especialización en Gestión Empresarial \\ juandavidgarro@gmail.com
}

\section{Resumen}

El propósito de esta investigación es identificar estrategias encaminadas a fortalecer la industria de desarrollo de software en Colombia; para lograr eso se utilizó el método francés de Michael Godet del análisis estructural prospectivo, y así se obtuvo una serie de tendencias con sus respectivas variables y comportamientos de las cuales se analizan el top 10 de mayor impacto. De este análisis se encontró que las empresas colombianas poseen la ventaja de ser veloces, agiles y eficientes para atender los rápidos, constantes y críticos cambios en todo el ciclo de vida del software.

Palabras clave: Análisis, Prospectiva, Software, Tendencias, Impactos. 
Introducción

La industria del desarrollo de software en todo el mundo ha crecido de tal manera que Colombia lo empieza a identificar como un como un renglón interesante de la economía nacional. De acuerdo con Martínez, Arango y Robledo (2015), desde comienzos de los años 90 esta industria se ha beneficiado por el ingreso de economías emergentes de otros países que brindan respuesta a un nicho de mercado determinado gracias a la generación de estrategias de desarrollo de software a la medida.

Si bien, las tendencias mundiales en muchos países desarrollados son los que jalonan y muestran el futuro de muchas de las tecnologías y maneras de hacer las cosas, en Colombia apenas se empieza a generar planes de trabajo que transforme el país en un consumidor digital desde diferentes industrias si no también como un exportador de este tipo de servicios.

Esta investigación refiere al análisis de las tendencias del país frente a diferentes puntos que enmarcan el macroentorno de las empresas de la industria del software, como son la política, la economía, el factor social, la tecnología, lo ecológico, y lo legal. Además de factores como clientes, proveedores, barreras de entrada, competencia, nueva competencia para identificar las oportunidades y amenazas que se generan desde estos aspectos y que las empresas pueden tomar para apalancar sus estrategias corporativas o por el contrario ser precavidos y prudentes al momento de la toma de decisiones.

La metodología usada fue el método francés de Michael Godet donde se permite realizar el análisis de las variables antes mencionadas y calificarlas por medio de una 
magnitud que denotara que tan impactantes es para la industria dicha variable, a partir de allí se realizan una serie de filtros los cuales ayudaran a determinar cuáles son variables tienen mayor dependencia e influencia sobre otras, para de este modo llegar claramente a reconocer cuales de los factores analizados tienen indecencia de factores externos a la empresa y esta puede tomar sus decisiones sobre ellos y cuales son necesariamente dependientes de factores externos, lo cual implica que queda por fuera del alcance la empresa para realizar cambios en sus condiciones.

Finalmente, desde el análisis de las variables del entorno, se identifican diferentes estrategias que fomentan el crecimiento de cada una de las compañías de la industria y que se implementan según la naturaleza, direccionamiento o necesidades propias de estas.

Análisis Prospectivo de la Industria de Desarrollo de Software en Colombia

El gobierno colombiano ha identificado la importancia de generar planes estratégicos que jalonen al país a un desarrollo económico y de productividad desde la industria del software y servicios TI que inclusive ayuden a mejorar la competitividad de otras. Según Ortega (2014), desde diferentes puntos el Estado trabaja en la transformación de la industria mediante el Plan Vive Digital, el cual le permitirá a los colombianos reducir las barreras que dificultan el acceso al uso de las TIC y se vuelva motor de desarrollo del país, generador de empleo y un renglón de la economía que fomenta la exportación de productos y servicios además de evolucionar el país a un consumo interno de economía digital. De acuerdo con Alarcón, Cañizares y Torres (2016), las TIC presenta diferentes oportunidades de soluciones, donde se incluyen tecnologías las cuales permiten el manejo 
y procesamiento de la información y de los datos. Por ello, una de las oportunidades en pro de este objetivo por parte del Ministerio de Tecnologías de la Información y las Comunicaciones (2019) es la ley 1341 de 2009 donde se impulsa el desarrollo y fortalecimiento del Sector de Tecnologías de la Información y las Comunicaciones, adicionalmente promueve investigación e innovación con el propósito de obtener competitividad y avance tecnológico en relación con el entorno nacional y extranjero. Donde a través de una evaluación de desempeño de la industria, un mapeo de instituciones tanto públicas como privadas, un análisis de las mejores prácticas a nivel internacional y la planeación a corto y mediano plazo permitan el crecimiento de manera conjunta de toda la economía del país, como se menciona en el informe del ministerio de Industria. Es importante entonces que las diferentes empresas de la industria sientan el avance y el respaldo del gobierno y la posibilidad de una economía en desarrollo y crecimiento.

Por otra parte, se encuentra el aspecto económico donde el crecimiento de la industria ha sido muy positivo, según Portafolio (2019) esta creció 19\% durante el 2018 lo cual se tradujo en un movimiento de 13.5 billones de pesos al año, que a su vez impactó en 1,7\% al PIB del país y donde las actividades se concentran en consultoría informática y desarrollo de software. Adicionalmente, en Latinoamérica se cuenta con una participación de $2.9 \%$ del gasto total de las TI del mundo, y Brasil es el que más gasto genera para este tema, tal como lo ha indicado Santos (2009). En entrevista con el diario económico Portafolio (2019) la directora de Fedesoft Paola Restrepo indicó que el ponderado de crecimiento de los últimos seis años es de $16.7 \%$, lo cual se espera que incremente con todos los planes que aportan al país. En la misma entrevista mencionó que esta es una 
industria que emplea a cerca de 115.000 personas en el sector en una plataforma de 7.650 empresas y los departamentos con mayor expansión son Cundinamarca, Antioquia y Valle. Adicionalmente, tal como lo menciona Piedrahita (2018), es una de las industrias donde más empresas se crean pues el $90 \%$ corresponde a micros y pequeñas empresas y gran porcentaje de ellas no tienen más de cinco años de constitución, lo cual genera cada vez más la oportunidad a la empleabilidad y al dinamismo a la economía del país. Y al considerar a Galves, Riascos, Contreras (2014), para las empresas el uso de las TIC facilita sus procesos y procedimientos al usar herramientas tecnológicas, las cuales reducen los costos existentes, generan oportunidades en el mercado y aumenta la probabilidad de éxito.

Como estrategia para el continuo crecimiento de la industria es importante el factor social donde las competencias y conocimientos específicos de la empresa y los empleados, es fundamental, y tal como lo mencionan Botello y Guerrero (2014), la competitividad de la industria aumenta y se vuelve llamativo para los mercados internacionales al momento de certificar en gran parte las empresas y los empleados de estas. Según el informe de Colombia Productiva (2017) en su encuesta a 330 empresas de la industria de aseguramiento a la calidad de software, $295(89,9 \%)$ no cuentan con ningún tipo de certificación como por ejemplo en Microsoft, ITIL, Oracle, Java. Esto es una oportunidad orientada a certificar la experiencia y el respaldo de la calidad de sus actividades laborales y profesionalismo en la materia. Es un punto importante para las empresas a nivel social crear estrategias entre sus empleados y procesos internos para cada vez reducir el porcentaje de empresas sin certificaciones de la industria. 
Similarmente el vínculo entre Universidad - Empresa - Estado constituye una oportunidad, en donde las garantías del gobierno para que cada vez más personas se gradúen de las universidades hacen que la mano de obra calificada pueda ocupar una vacante rápidamente. Portafolio (2019) indica que en Colombia existen más de 200 facultades de Ingeniería de Sistemas y estas no son suficientes para la demanda actual y que a su vez, permitan una mejora en la industria. Por su parte, el Ministerio de Educación (2019) resalta que este Vínculo es soporte institucional para la innovación como también el fortalecimiento del capital humano, la inversión en laboratorios científicos y apoyos a proyectos de Investigación los cuales impulsan a las regiones. Es importante que esta triada este en constante evolución y sinergia para poder ofrecer oportunidades y crecimiento hacia las tres dimensiones. Pues el estudiante encontrara donde realizar sus prácticas y donde poder desenvolverse como profesional al ejecutar toda su capacidad académica e investigativa y realizar carrera como profesional, mientras que las empresas podrán ocupar las vacantes que necesitan con personal calificado y además contaran con el respaldo de las universidades que los pueden orientar sobre sus desafíos más grandes en la industria.

Como otra oportunidad, según Colciencias, los Parques Científicos, Tecnológicos y de Innovación son entornos que promueven la transferencia de conocimiento y tecnologías en pro del desarrollo regional actualmente se encuentran en etapa de implementación - Piloto 2016-2020 y se estima que para el periodo 2021-2026 se consolide una etapa de crecimiento y a partir del 2026 la expansión permita apoyar las estabilizaciones de todas aquellas iniciativas y apuntar a la internacionalización de esta estrategia. Conforme con Colciencias (2019), dentro de los Parques más reconocidos se 
encuentran: Parque Biopacífico, Guatiguará, Tecnológico de Bogotá, ParqueSoft, Ruta N, Parque Explora, Parque E y pese a su existencia, estos clústeres de Ciencia y Tecnología Informática aún no son suficientes para responder a las necesidades del país, de allí el compromiso del Gobierno Nacional para realizar una expansión en 8 años aproximadamente, con los recursos y capacidades para estar a la vanguardia en la generación de innovación y la revolución tecnológica.

Desde el componente ecológico, la sostenibilidad de la industria se presenta como una oportunidad, la conciencia ambiental de la industria del desarrollo de software busca disminuir el volumen y transferencia de datos en los servidores, al contribuir a la disminución de emisiones de carbono, así mismo como lo indica Aranda Software (2019), el desarrollo de software eficiente permite disminuir el consumo energético y los datos rasterizados en un 95\%. Gil, Fernández, García, Luján y Toval (2018) estiman que para el año 2020, las emisiones de dióxido de carbono y generadas por las TICs, representarán un $3 \%$ a nivel global.

Conforme a Plataforma Ambiental Data (2019) el estudio del Medio Ambiente implica entre otras cosas, "el intento de comprender, modelar y de cierta forma predecir los procesos que ocurren en la naturaleza y su relación con las actividades humanas" y a través del desarrollo de software, se brindan herramientas para apoyar dichas labores; actualmente se pueden utilizar para determinar la calidad del aire y de agua, realizar investigaciones sobre biodiversidad y ecosistemas, analizar datos geoespaciales, entre otros. Según el periódico El Espectador (2015), en Colombia existen iniciativas que promueven el cuidado del medio ambiente, por ejemplo, Tremarctos, es una herramienta 
que se desarrolló para realizar trazados viales y amigables con el medio ambiente o en la ejecución de proyectos mineros, petroleros, energéticos y agropecuarios que cuiden el medio ambiente y todas las especies que se encuentran en peligro de extinción.

Desde el Ministerio de Comercio, Industria y Turismo en el año 2008, como oportunidad se crea el Programa de Transformación Productiva cuya finalidad está dirigida a impulsar la productividad y competitividad de las diferentes industrias de Colombia; así mismo ayuda a cumplir las metas propuestas en la Política Nacional de Competitividad y Productividad (Conpes 3527 de 23 de junio de 2008) y actualizada por la Política de Desarrollo Productivo (Conpes 3866 de 20016). Sus acciones están enmarcadas en dos objetivos específicos: mejorar la productividad y orientar a las empresas a que tengan la capacidad para usar y disfrutar de los acuerdos comerciales vigentes en el país para hacer más fácil la comercialización de productos, relaciones con clientes y proveedores.

Hoy en día la tendencia tecnológica avanza a una velocidad alta y proporciona nuevas y mejores soluciones para las personas, la innovación y las invenciones están a la vuelta de la esquina, grandes compañías hoy trabajan en esto y el mercado lo exige, por lo que se debe estar preparado y así mismo ser competitivo pues la exigencia es alta y los resultados son inmediatos, lo que hoy fue inventado mañana ya será obsoleto. En el mundo actual se trabaja en diferentes campos: es el caso de la Inteligencia Artificial (AI), la cual dependerá de la combinación de software con datos y programación; el Bitcoin el cual es dinero digital y que será el dinero futuro de las personas; el internet de las cosas IoT donde su finalidad es conectar los objetos al internet; aplicaciones inteligentes las cuales se adaptan a la necesidad de las personas; Bigdata capta información importante para las 
empresas; Robótica donde en un futuro se puede remplazar la mano de obra de las personas por robots; la Biotecnología la cual ayuda en el desarrollo de necesidades humanas, según el portal Infobos (s.f.). Por otra parte, se encuentra que la tecnología de la información y comunicaciones conectadas con las finanzas ya sea de personas o corporativas, brindan oportunidades de cambios y un nuevo rol a las organizaciones como lo indican Sánchez, Sánchez y López (2016).

En este sentido, un factor de alto impacto que no debe olvidarse son las vulnerabilidades del software que como lo refiere Monster (2019), son falencias que exponen debilidades a la ciberseguridad de los dispositivos de la red y que amenaza la industria, por lo tanto el componente de seguridad informática debería ser un plus que cada día tome más fuerza, como factor diferencial al promover las políticas de seguridad de la información y ciberseguridad para la incorporación de software en las diferentes organizaciones, así como para su gestión, dirección, monitoreo, mantenimiento y control, al considerar esquemas técnicos, procedimentales, metodológicos y de política nacional tal como se refiere el ministerio de las Tics. Esto último, como fundamento expresado por MINTIC (2014).

Desde lo sectorial y de acuerdo con el portal Achilles (s.f.), es claro que los proveedores son parte de las compañías, pues estos son fundamentales para el funcionamiento y el desarrollo de un producto o servicios en la medida que las empresas cuentan con un insumo constante, por ello debe realizarse un seguimiento al desempeño de estos y así mismo tener muy claros los acuerdos de servicio. Por su parte, la relación que se maneje con estos posibilita en gran medida que las partes lleguen a un gana-gana dentro 
de la negociación. De acuerdo con los datos entregados por la APC Colombia y la UNOSSC (2018) en lo referente a la competencia, durante los últimos 5 años la industria de software en Colombia ha crecido $16 \%$ y se pronostica un $19 \%$ en el 2019. Por otra parte, en el 2018 se estimaba un aproximado de 7000 empresas de software en donde el $82 \%$ corresponde a microempresas, $13 \%$ pequeñas, $3 \%$ medianas y solo el $2 \%$ a grandes.

Actualmente, se cuentan con un número representativo de compañías dedicadas a la prestación de servicios en la creación, desarrollo e innovación de sistemas de software, especializadas en sectores específicos en el mercado del país, enfocadas en mayoría a actividades de información y comunicación y en una minoría a las actividades que tienen relación con la educación. La demanda actual se ha elevado debido al crecimiento de la infraestructura relacionada con la tecnología y a la alta calidad en la elaboración de sus productos. Pese a ello, existe una amenaza de barreras de entrada en relación con el índice de rotación de personal que existe en la industria. Según entrevista a la directora de Fedesoft Paola Restrepo para el diario Portafolio, menciona que existe un déficit de 62.000 personas y que la rotación de personal se encuentra en 26,45\%, lo cual lo hace una razón importante para tener en cuenta en todas aquellas compañías que consideran ingresar, impulsar o sostenerse en la industria. Sin embargo, no hay que desconocer que el ingreso de las compañías extranjeras al país también activan la dinámica de los competidores dentro de la industria, esto como amenaza al ingresar con mejores tecnologías, más garantías en cuestiones salariales, de acuerdo con ProColombia, entre agosto de 2018 y julio de 2019, ingresaron más de 185 proyectos de inversión extranjera que dinamizan la economía, entre ellos 23 pertenecientes a Software y TI; de acuerdo con el diario La 
República (2019), en total el país recibió US\$6.532 millones y se destaca la estrategia del gobierno para atraer dicha inversión con incentivos tributarios.

Además, al considerar lo planteado por la Revista SG (s.f.), Colombia tiene una gran oportunidad dentro de la industria al contar con un Ministerio dedicado a las Tecnologías de la Comunicación, MinTIC, que se encarga de diseñar, adoptar y promover las políticas, programas y proyectos de este sector, que apunta a una transformación para la industria cuyos esfuerzos incentivan las compañías dedicadas al software para que estas sean más competitivas, generen empleo, y al mismo tiempo fortalece aquellas que ya existen y aportan al desarrollo económico del país, como se menciona en el portal del Ministerio de TIC (2019).

\section{Metodología}

El desarrollo y profundización de la investigación se dio a partir de la propuesta realizada de analizar prospectivamente la industria del software en Colombia, para esto se evaluaron 16 variables de la cuales 8 corresponden al macroentorno y 8 al sectorial. El macroentorno fue analizado bajo la herramienta PESTEL, la cual ayuda a realizar un análisis prospectivo del entorno e identificar amenazas u oportunidades dentro de los factores Político, Económico, Socio Cultural, Ecológico y Legal. Las 8 variables restantes se analizan sectorialmente bajo el principio de las 5 fuerzas de Porter cuya finalidad es mejorar los recursos y aumentar la competitividad 
Para cada una de las variables analizadas, se describe brevemente con cifras y datos de acuerdo con las fuentes de investigación, posteriormente se analiza su clasificación en Oportunidad o Amenaza ya que son variables externas a la empresa. Se determina la magnitud del impacto en una escala de 0 a 3 ( 0 - nulo, 1 - bajo, 2 - medio, 3 - alto) y para obtener el top 10, se elabora un ranking de las variables de acuerdo con el impacto resultante del paso anterior tal como se observa en la Tabla 1 en los Anexos. Con el recurso proporcionado por el docente en el aula de clase "Software Prospectiva.xlsm", el ranking fue calculado automáticamente en posiciones de la 1 a la 10 . Con estas 10 variables más significativas se realiza la matriz de impactos cruzados (ver Tabla 2, Anexos), la cual permite medir la interdependencia entre diversos factores clave, primero se analiza la dependencia que tiene cada una de las variables y luego la influencia que tiene esta frente a cada una.

Finalmente, la herramienta de forma automática calcula el análisis MIC-MAC, un análisis estructural, el cual tiene como finalidad ubicar las variables analizadas en los impactos cruzados en una zona de poder o de conflicto que tengan directa relación de forma influyente o dependiente de cada una de ellas. Con el resultado que se obtiene, se proponen estrategias para la industria de desarrollo de software en Colombia.

\section{Resultados Obtenidos}

Mediante el método de análisis estructural aplicado en el estudio de esta investigación se logró clasificar y describir las variables más relevantes y determinantes de la industria del Software en Colombia, la cual brinda oportunidades en la generación de 
estrategias que ayuden a mitigar aquellos factores de riesgo que son incontrolables por la compañía, además de potencializar aquellos que tienen un alto grado de influencia y dependencia para el desarrollo de esta industria.

El resultado que arrojó después de aplicar la metodología propuesta y de analizar las 16 variables fueron 9 variables, las cuales quedan catalogadas de la siguiente manera: una variable en el cuadrante I (Zona de Poder) y las restantes en el cuadrante II (Zona de conflicto). Este resultado puede observarse en la Ilustración 1:
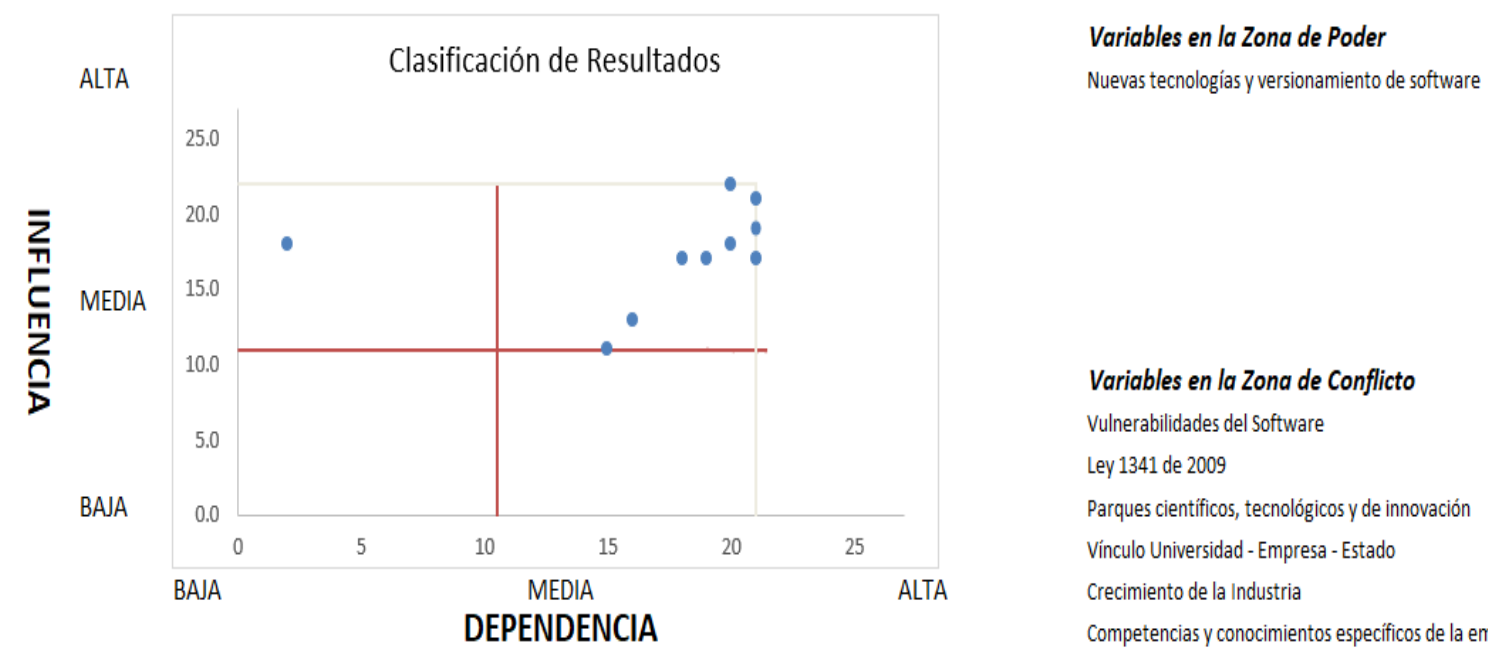

Implementación de software en pro de la conservación y cuidado del medio ambiente

\section{Ilustración 1 Gráfico MIC-MAC}

Fuente: Elaboración propia con base en el aplicativo de prospectiva del Politécnico Grancolombiano.

En el cuadrante I (Zona de Poder), la variable que ejerce una alta influencia e impacto, de la que dependerá y que se puede fortalecer para el futuro de la industria son las Nuevas Tecnologías y Versionamiento de Software que realizan las compañías 
mediante la innovación, y la incorporación de nuevas metodologías para el diseño, desarrollo y verificación de calidad en la creación de sus programas, ya que como se observa en los indicadores del informe de la Superintendencia de Sociedades (2015), entre el periodo 2010-2014 la tasa de crecimiento anual compuesta ha crecido un $36 \%$, lo cual muestra cada vez más la demanda de consumo por servicios de desarrollo de software. Por lo cual una de las estrategias que deben implementarse para el sostenimiento y la expansión tanto a nivel nacional como internacional de esta variable, se enfoca en el desarrollo de nuevas soluciones de software donde se generen innovaciones disruptivas que se adelanten las necesidades del mercado, y rápidamente dejan otras versiones obsoletas lo cual estimula la demanda. Según Sáenz, Gutierrez y Ramos (2018), el desarrollo de cualquier producto de software no termina con la entrega de producto final, existe un periodo de soporte entre 2 a 3 años de actualizaciones y mejoras continuas para evitar la curva de obsolescencia.

Finalmente, en el cuadrante II (Zona de Conflicto), se observan aquellas variables que generan un alto riesgo, las cuales conllevan un gran impacto, debido a que estas son incontrolables internamente por las compañías, que dependen e influyen en el desarrollo del software en Colombia por lo que se consideran de alta importancia para la industria. Después de realizar la metodología del análisis estructural esta arrojó las siguientes 8 variables claves que muestran el mayor impacto:

1. Vulnerabilidades del Software.

2. Ley 1341 de 2009.

3. Parques científicos, tecnológicos y de innovación.

4. Vínculo Universidad - Empresa - Estado. 
5. Crecimiento de la Industria.

6. Competencias y conocimientos específicos de la empresa y los empleados.

7. Sostenibilidad de la Industria.

8. Implementación de software en pro de la conservación y cuidado del medio ambiente.

Como se puede evidenciar en el desarrollo de este trabajo, la industria del software en el país cuenta con un gran potencial para el futuro, sin embargo, depende de una serie de variables incontrolables por las empresas, lo cual hace muy sensible a cualquier cambio radical que surja en el macroentorno de estas. Las organizaciones de esta industria pueden implementar y fundamentar un enfoque prospectivo cuya actitud y acción proactiva provoquen que las cosas sucedan; el seguimiento a las variables expuestas y a los cambios que pueden ofrecer o amenazar su entorno permiten establecer una ruta donde las fortalezas se construyen, las oportunidades se buscan, las debilidades se prevén y las amenazan se reconocen. Por ello, se plantean las siguientes estrategias:

Diversificación concéntrica. Según Pérez Moya (1996) esta estrategia está dirigida a incluir más productos o servicios adicionales a los que ya se tienen con el fin de ingresar a nuevos mercados. La industria puede incorporar nuevos productos o servicios de forma integral, por ejemplo: mantenimiento de software, inteligencia artificial, big-data o ciencia de datos, inteligencia de negocios, administración del riesgo en componentes dependientes de software, entrenamiento y formación, creatividad e innovación en la solución de problemas y en toma de decisiones, automatización robótica de procesos, entre otros. Lo anterior, es una oportunidad desde cada compañía para que fortalezca el área de 
I+D, construya nuevas fortalezas al consolidar las actuales y disminuir las debilidades y contrarrestar las variables de parques científicos, tecnológicos y de innovación, vínculo Universidad - Empresa - Estado, competencias y conocimientos específicos de la empresa y los empleados, que se encuentra en la zona de conflicto.

Las alianzas estratégicas. Estas son estrategias que fortalecen el crecimiento y la sostenibilidad de la industria, como también, la implementación de software en pro de la conservación y cuidado del medio ambiente. Según Gray (s.f.), la alianza estratégica es una "relación entre dos o más entidades que acuerdan compartir los recursos para lograr un objetivo de beneficio mutuo", en este sentido, las empresas de la industria pueden realizar alianzas de tal manera que mejoren el valor percibido de su oferta actual al ofrecer productos o servicios de otras compañías que puedan estar dirigidos a clientes similares, lo cual ayuda en reforzar su posicionamiento del negocio y de su marca. Una de las posibles alianzas es la coalición, según la Organización Internacional del Trabajo (s.f.), las empresas colaboran entre si con el objetivo de lograr metas en común; por ello, desde la alta gerencia se pueden realizar acuerdos de cooperación para el impulso del desarrollo tecnológico con otras compañías, así mismo acceder o incursionar en aquellas economías de grande escala donde se podrá obtener más conocimiento, tecnología de punta e innovaciones con alto desempeño, que a su vez cubre el riesgo de obsolescencia de los productos. todo lo anterior lleva a la empresa a explorar nuevos mercados con más confianza y con una oferta de productos y servicios más amplia y de mejor calidad, situación que deja a la compañía en un punto de competencia más alto frente a la oferta de la industria. Por ello como estrategia se recomienda implementar la coalición de marcas y de tecnologías. 
Para implementar las estrategias de coalición se debe considerar un análisis de requisitos como son: las posibles empresas para realizar la alianza, el alcance y el tiempo propuesto para cumplir los objetivos, además de los recursos financieros, tecnológicos y de capacidad humana que se comparten y la distribución de utilidades y rentabilidades.

Como coalición tecnológica una manera de unirse es que las empresas se encuentren sobre la misma línea de mercado, por ejemplo, fábricas de software con empresas de aseguramiento de la calidad del software, empresas de ciberseguridad con empresas de certificaciones en seguridad informática, entre otras.

Por otro lado, la coalición de mercado, como alternativa puede orientar a especializarse en un sector por ejemplo el bancario, video juegos, manejo de datos, entre otros. De esta manera las empresas que se unan podrán sacar provecho de oportunidades de negocio más ventajosa, un banco podrá siempre contar con los servicios de una empresa especialista que si bien la primera no hace parte de la industria del software cuenta con el respaldo de la empresa especialista, ambas ganaran reputación, respaldo y la empresa de software podrá ganar más mercado al atender empresas con el mismo tipo de necesidades.

\section{Conclusiones}

La Industria de Software en Colombia ha evolucionado y se ha convertido en uno de los líderes en América latina en innovación, desarrollo y alta calidad en sus productos de software, lo que ha generado que las empresas sean más competitivas y eficientes, al generar la oportunidad de nuevos empleos y de esta forma mitigar de forma gradual la crisis de desempleo que existe en el país. Esto concuerda con Toro y Peláez (2018), que 
establecen que uno de los pilares estratégicos organizaciones es la calidad en el desarrollo y mantenimiento del software.

Debido a los constantes cambios a nivel tecnológico dentro la industria se ve la necesidad en que las compañías dedicadas al software deben enfocarse diariamente a la producción de nuevos productos y servicios, además de capacitar a todos los colaboradores y estar así a la delantera en la transformación del mundo digital, pues estos dos factores ayudan a las compañías a posicionarse a nivel nacional e internacional, por lo que las conducirán al aprovechamiento de sus recursos y al mismo tiempo al sostenimiento, incremento en su productividad y en empresas mucho más eficientes.

Las entidades gubernamentales en pro de impulsar la industria del software se han propuesto incorporar estrategias y beneficios tanto económicos, aduaneros y tributarios para aquellas nuevas compañías que decidan incorporarse y para las ya existentes expandirse a nivel internacional y de esta manera contribuir tanto a la economía de la compañía como la del país, al enfocar de esta manera los esfuerzos en temas de innovación, investigación y factor social, al ofrecer salarios competitivos, retención de conocimiento, y disminución en la rotación de personal, al ser el factor humano uno de los activos más relevantes dentro de las organizaciones.

Para disminuir las afectaciones ambientales que se generan en la industria del software, se hace necesario implementar procesos y políticas que disminuyan este impacto, según Radu (2018) esto se logra al realizar un uso adecuado y responsable de los equipos y al mismo tiempo reutilizándolos en un nuevo y mejorado software que evite el constante 
reproceso de mano de obra y que logre aumentar la eficiencia de la compañía mediante una mejora continua.

La industria del Software al estar expuesta a la imposición de cambios incontrolables por parte externa de las compañías genera un grado de incertidumbre la cual la convierte en una variable que se debe de tener en cuenta al momento de una negociación contractual con clientes y proveedores, para que de esta manera se pueda generar de una forma estratégica en aras de no perjudicar a futuro el desarrollo de un servicio o producto y que posteriormente perjudique las utilidades de la compañía. Adicionalmente, las empresas con propósitos multiservicios, adquieren la característica de que, si no obtienen buenos resultados en alguno de ellos, se puede amortiguar dicho fracaso el éxito de los demás servicios.

En el gráfico MIC-MAC, las variables del entorno que quedan sobre la zona de poder son aquellas donde la empresa puede invertir sobre $\mathrm{I}+\mathrm{D}+\mathrm{i}$ al tener en cuenta el crecimiento y el buen respaldo a la industria, mientras otras variables identificadas sobre la zona de conflicto son exógenas a la empresa, pero valdrá la pena analizarlas cada cierto tiempo para validar los escenarios y tendencias que se marcan.

La diversificación concéntrica, las alianzas estratégicas, las coaliciones, hacen parte de las estrategias más confiables para ser implementadas en las empresas de software, pues los rápidos y constantes cambios implican una respuesta veloz, ágil y eficiente, de tal manera que se reduzcan los fallos y las situaciones de emergencia en el ciclo de vida del software, así mismo la existencia de un mejoramiento continuo en los procesos 
importantes, críticos o ineficientes, y finalmente la respuesta proactiva ante las circunstancias de la industria. 
Referencias

Achilles. (s.f.). Achilles. Obtenido de La importancia de la gestión de proveedores: https://www.achilles.com/es/industry-insights/la-importancia-de-la-gestion-deproveedores/

Alarcón, R. V., Cañizares Stay, A., \& Torres Saybay, E. G. (2016). Software Libre en Las Empresas. Caribeña de Ciencias Sociales.

APC-Colombia y UNOSSC. (2018). Colombia Cambió Innovaciones para el Desarrollo. Obtenido de https://issuu.com/apc-colombia/docs/colombia_cambio_final

Aranda Software. (Septiembre de 2019). El software también puede ser sostenible. Obtenido de https://arandasoft.com/el-software-tambien-puede-ser-sostenible/

Botello Peñaloza, H. A., \& Guerrero Rincón, I. (2014). Condiciones y determinantes de la internacionalización de las empresas industriales colombianas: una aproximación cuantitativa en el ámbito de la firma. Apuntes del Cenes vol.33 no.57, http://www.scielo.org.co/scielo.php?script=sci_arttext\&pid=S012030532014000100009.

Colciencias. (18 de Mayo de 2016). Obtenido de https://www.colciencias.gov.co/sites/default/files/upload/noticias/presentacioncolciencias-parques.pdf

Colciencias. (Agosto de 2019). Parques Científicos, Tecnológicos y de Innovación PCTI. Obtenido de https://www.colciencias.gov.co/reconocimiento-actores/parquescientificos-tecnologicos-y-innovacion-pcti

Colombia Productiva. (Marzo de 2017). Plan de Negocios 2017. Obtenido de Plan de Acción a Corto y Mediano Plazo Sector Software y Servicios TI: https://www.colombiaproductiva.com/ptpcapacita/publicaciones/sectoriales/publicaciones-software-y-ti/plan-de-negocios2017

Cuenca Díaz, C. (Agosto de 2019). Desarrollo Seguro: Principios y Buenas Prácticas. Obtenido de https://www.owasp.org/images/9/93/Desarrollo_Seguro_Principios_y_Buenas_Pr $\%$ C3\%A1cticas..pdf

El Espectador. (Septiembre de 2015). Tecnología para el ambiente. Obtenido de https://www.elespectador.com/especiales/tecnologia-el-ambiente-articulo-586815

Gálvez Albarracín, E. J., Riascos Erazo, S. C., \& Contreras Palacios, F. (2014). Influencia de las tecnologías de la información y comunicación en el rendimiento de las micro, pequeñas y medianas empresas colombianas. Estudios Gerenciales, 355-364.

Gil, D., Fernández, J. L., Trujillo, J., García Mateos, G., Luján Mora, S., \& Toval, A. (2018). The Effect of Green Software: A Study of Impact Factors on the Correctness of Software. Sustainability, 1-19.

Gray, C. (s.f.). Qué son las alianzas estratégicas. Obtenido de La Voz de Houston: https://pyme.lavoztx.com/qu-son-las-alianzas-estratgicas-8587.html 
Infobos. (s.f.). Obtenido de 7 tecnologías que arrasarán en 2019: https://orientacionlaboral.infojobs.net/nuevas-tecnologias-busqueda-empleo

La Republica. (agosto de 2019). Obtenido de Agroindustria y software concentran los proyectos de inversión extranjera directa:

https://www.larepublica.co/economia/agroindustria-y-software-concentran-losproyectos-de-inversion-extranjera-directa-2897745

Martínez Marín, S. J., Arango Aramburo, S., \& Robledo Velásquez, J. (2015). El Crecimiento de la Industria del Software en Colombia. Obtenido de Revista EIA: http://www.scielo.org.co/scielo.php?script=sci_arttext\&pid=S1794$12372015000100009 \& \operatorname{lng}=$ en\&tlng=es

Ministerio de Educación. (2019). Vínculo Universidad - Empresa - Estado. Obtenido de https://www.mineducacion.gov.co/1621/w3-printer-232769.html

MINTIC. (Marzo de 2014). Agenda Estratégica de Innovación: Ciberseguridad. Obtenido de https://www.mintic.gov.co/portal/604/articles-6120_recurso_2.pdf

MINTIC. (2019). Dirección de Desarrollo de Industria TI. Obtenido de https://www.mintic.gov.co/portal/604/w3-propertyvalue-557.html?_noredirect=1

Monster. (Agosto de 2019). Amenazas Informáticas. Obtenido de https://www.monster.es/orientacion-laboral/articulo/amenazas-informaticas

Organización Internacional del Trabajo. (s.f.). Guía Práctica: Entorno Propicio para las Empresas Sostenibles. Obtenido de http://eesetoolkit.itcilo.org/images/stories/pdf/spanish/sp_29_part3_tool7.pdf

Ortega Ruiz, C. A. (2014). Inclusión de las TIC en la empresa colombianaIncorporation of ITC into Colombian businesses. Suma de Negocios, 29-33. Obtenido de https://www.sciencedirect.com/science/article/pii/S2215910X14700060

Pérez Moya, J., \& Pérez Moya, J. (1996). Estrategia, gestión y habilidades directivas: un manual para el nuevo directivo. Madrid: Ediciones Díaz de Santos.

Piedrahita, E. (Octubre de 2018). La clave es el 'software'. Obtenido de Semana: https://www.semana.com/opinion/articulo/industria-del-software-en-colombiacolumna-de-esteban-piedrahita/587171

Plataforma Ambiental Data. (Septiembre de 2019). Software libre para estudios ambientales. Obtenido de https://ambientaldata.com/informacion/software-librepara-estudios-ambientales/

Portafolio. (Mayo de 2019). Obtenido de 'Debemos mejorar los índices de rotación de personal en sector TIC': https://www.portafolio.co/economia/empleo/debemosmejorar-los-indices-de-rotacion-de-personal-en-sector-tic-529996

Radu, L. D. (2018). An Ecological View on Software Reuse. Informatica Economica, 7585.

Sáenz Blanco, F., Gutiérrez Sierra, F., \& Ramos Rivera, J. C. (2018). Conformación de Equipos Ágiles para el Desarrollo de Software: Revisión de Literatura. Dimensión Empresarial, 39-54.

Sánchez Parrales, L. A., Sánchez Parrales, C. E., \& López Coloma, R. V. (2018). Las TICs en las Finanzas. Caribeña de Ciencias Sociales.

Santos Hernández, V. (2009). La Industria del Software, Estudio a Nivel Global y América Latina. Revista Académica de Economía Latinoamericana. 
SG. (s.f.). Obtenido de La Industria de Software en Colombia: https://sg.com.mx/revista/50/la-industria-software-colombia

Superintendencia de Sociedades. (2015). Desempeño del Sector Software 2012-2014. Bogotá: Delegatura para Asuntos Económicos y Contables Grupo de Estudios Económicos y Financieros.

Toro, A., \& Peláez, L. E. (2018). Validación de un modelo para el aseguramiento de la calidad del software en MIPYMES que desarrollan software en el Eje Cafetero. Entre Ciencia e Ingeniería, 84-92.

Velneo. (enero de 2019). Obtenido de El desarrollo de software de gestión en Colombia en 2019: https://velneo.es/el-desarrollo-de-software-de-gestion-en-colombia-en2019/ 